\title{
Análise da educação inclusiva como temática de pesquisa nos programas de pós- graduação stricto sensu do Instituto Federal do Triângulo Mineiro - IFTM
}

Analysis of inclusive education as a research subject in stricto sensu postgraduate programs of the Federal Institute of Triangulo Mineiro - IFTM

Análisis de la educación inclusiva como sujeto de investigación en los programas de posgrado estricto sensu del Instituto Federal del Triangulo Mineiro - IFTM

Recebido: 06/09/2021 | Revisado: 12/09/2021 | Aceito: 14/09/2021 | Publicado: 16/09/2021

\author{
Luisa Helena de Almeida Ribeiro \\ ORCID: https://orcid.org/0000-0001-5807-5599 \\ Instituto Federal de Educação, Ciência e Tecnologia do Triângulo Mineiro, Brasil \\ E-mail: luisaribeiro@iftm.edu.br \\ Rosemar Rosa \\ ORCID: https://orcid.org/0000-0002-2048-9195 \\ Instituto Federal de Educação, Ciência e Tecnologia do Triângulo Mineiro, Brasil \\ E-mail: rose@iftm.edu.br
}

\begin{abstract}
Resumo
O presente estudo objetivou analisar as pesquisas sobre a temática Educação Inclusiva em programas de Pósgraduação Stricto Sensu do IFTM, investigar as disposições sobre a Educação Inclusiva estabelecidas na Lei N ${ }^{\circ}$ 13.146, de 6 de julho de 2015 e verificar em quais programas de Pós-graduação Stricto sensu do IFTM são desenvolvidas pesquisas sobre o tema. A metodologia envolveu pesquisas bibliográfica e documental no Catálogo de Teses e Dissertações do IFTM, com o propósito de levantar os dados necessários para responder ao objetivo geral do presente trabalho. Os resultados obtidos demonstraram que a maioria das pesquisas foram desenvolvidas no Mestrado Profissional em Educação Tecnológica (PROFEPT), sendo um trabalho focado na inclusão social e profissional; outro apresentou uma análise da contribuição das Tecnologias de Informação e Comunicação (TIC) para a aprendizagem; um estudo desenvolveu um aplicativo móvel para auxiliar crianças autistas em seu cotidiano e a última pesquisa selecionada referente ao Núcleo de Atendimento às Pessoas com Necessidades Educacionais Específicas (NAPNE) do IFTM. Conclui-se que, dada a importância do assunto e o reduzido número de estudos na área, torna-se necessário o incentivo de novas pesquisas, bem como o acompanhamento dos trabalhos já desenvolvidos, pois as experiências socializadas configuram estratégias para o alcance dos objetivos da Educação Inclusiva.
\end{abstract}

Palavras-chave: Educação inclusiva; Pós-graduação Stricto Sensu; Dissertações.

\begin{abstract}
This study aimed to analyze research on the theme of Inclusive Education in Stricto Sensu Graduate Programs at the IFTM, investigate the provisions on Inclusive Education established in Law No .13 .146 , of July 6, 2015 and verify in which Graduate programs. Stricto sensu graduation from the IFTM researches are developed on the subject. The methodology involved bibliographic and documentary research in the IFTM Theses and Dissertations Catalog, with the purpose of raising the necessary data to respond to the general objective of the present work. The results obtained showed that most of the researches were developed in the Professional Master's Degree in Technological Education (PROFEPT), being a work focused on social and professional inclusion; another presented an analysis of the contribution of Information and Communication Technologies (ICT) to learning; a study developed a mobile application to help autistic children in their daily lives and the last selected survey was related to the IFTM's Center for Assistance to People with Specific Educational Needs (NAPNE). It is concluded that, given the importance of the subject and the small number of studies in the area, it is necessary to encourage further research, as well as the followup of work already developed, as socialized experiences configure strategies to achieve the goals of Inclusive education.
\end{abstract}

Keywords: Inclusive education; Graduate Stricto Sensu; Dissertations.

\section{Resumen}

El presente estudio tuvo como objetivo analizar la investigación sobre el tema de Educación Integrada en los programas de Posgrado Stricto Sensu de la IFTM, investigar las disposiciones sobre Educación Integrada establecidas en la Ley $\mathrm{N}^{\circ} 13.146$, de 6 de julio de 2015 y verificar en qué programas de Posgrado. Stricto sensu egresados de la IFTM se desarrollan investigaciones sobre el tema. La metodología involucró la investigación bibliográfica y 
documental en el Catálogo de Tesis y Disertaciones de IFTM, con la finalidad de recabar los datos necesarios para dar respuesta al objetivo general del presente trabajo. Los resultados obtenidos mostraron que la mayoría de las investigaciones se desarrollaron en la Maestría Profesional en Educación Tecnológica (PROFEPT), siendo un trabajo enfocado a la inclusión social y profesional; otro presentó un análisis de la contribución de las tecnologías de la información y la comunicación (TIC) al aprendizaje; un estudio desarrolló una aplicación móvil para ayudar a los niños autistas en su vida diaria y la última encuesta seleccionada estaba relacionada con el Centro de Asistencia a Personas con Necesidades Educativas Específicas (NAPNE) del IFTM. Se concluye que, dada la importancia del tema y el escaso número de estudios en el área, es necesario incentivar una mayor investigación, así como el seguimiento de los trabajos ya desarrollados, ya que las experiencias socializadas configuran estrategias para lograr los objetivos. de educación inclusiva.

Palabras clave: Educación inclusiva; Licenciado Stricto Sensu; Disertaciones.

\section{Introdução}

O tema Educação Inclusiva ganhou destaque a partir dos anos 90, com as promulgações de políticas públicas nacionais e internacionais que favoreceram a oferta do ensino às pessoas com deficiência. Desde então estudos vêm sendo realizados com o objetivo de promover a inclusão nos ambientes escolares e identificar melhores práticas e ações que favoreçam esse trabalho.

Vários estudos, a exemplo de Santos (2011), Menezes (2015) e Gonçalves (2018), expõem estratégias para a consecução dos objetivos das políticas de inclusão ao permitirem conhecer a vivência dessas políticas e da aplicação das normativas, além de possibilitar a manifestação das percepções dos sujeitos envolvidos. Além disso, as produções científicas permitem o mapeamento de regiões e a destinação de investimentos, treinamentos e ações favoráveis às práticas estudadas.

Diante do exposto, o presente trabalho tem como objetivo geral analisar as pesquisas sobre a temática educação inclusiva, em programas de Pós-graduação Stricto sensu do Instituto Federal do Triângulo Mineiro (IFTM), sendo a Educação Inclusiva compreendida, nesse contexto, como um conjunto de ações e estratégias de inserção de indivíduos com qualquer tipo de deficiência dentro do ambiente escolar regular. (Aranha, 2005).

O estudo tem ainda como objetivos específicos investigar as disposições sobre a Educação Inclusiva estabelecidas na Lei Brasileira de Inclusão da Pessoa com Deficiência (LBI) - Estatuto da Pessoa com Deficiência, lei Nº 13.146, de 6 de julho de 2015 (BRASIL, 2015) e verificar em quais programas de Pós-graduação Stricto sensu do IFTM são desenvolvidas pesquisas sobre Educação Inclusiva.

A justificativa para a pesquisa embasa-se na representatividade que os estudos sobre Educação Inclusiva têm, de forma geral, na vida das pessoas com deficiência, sendo possível classificar essas pesquisas como estratégias para a promoção da qualidade de vida e trabalho, pois ações nesse sentido promovem interesse e divulgação dos recursos e serviços disponíveis. (Galvão Filho, 2013). Além disso, essas pesquisas atendem a orientação da LBI, que estabelece, no seu art. 77, que o poder público deve fomentar o desenvolvimento, a pesquisa, a inovação e a capacitação tecnológica, voltados para inclusão social.

A Educação Inclusiva pode ser entendida como uma abordagem contemporânea ao ensino que tem como objetivo assegurar a todos o direito à educação, por meio da presunção de igualdade, de oportunidades e do respeito às diferenças humanas, atentando, assim, às diversidades sociais, étnicas, culturais, físicas, intelectuais, sensoriais das pessoas. Seu principal objetivo é identificar e suprir as necessidades educativas especiais de todos os alunos com necessidades educacionais especiais, em salas de aula comuns do sistema regular de ensino, de forma a estimular a aprendizagem e o desenvolvimento pessoal e acadêmico de todos, buscando ainda a implantação de uma cultura de respeito nas práticas e políticas vigentes na escola e nos sistemas de ensino. (Mantoan, 2004).

Na década de 90, a Educação Inclusiva ganhou destaque mundial ao ser alvo de discussões e argumentações em grandes encontros sobre o tema, como por exemplo, a Conferência Mundial sobre Educação para Todos (Jomtien, Tailândia 1990), a Conferência Mundial sobre Necessidades Educacionais Especiais (Salamanca, Espanha - 1994) e a Convenção Interamericana para a Eliminação de Todas as Formas de Discriminação contra as Pessoas Portadoras de Deficiência (Guatemala 
- 1999). A partir desses encontros vários países passaram a estimular a implantação da Educação Inclusiva por meio da elaboração de políticas públicas para o seu desenvolvimento.

O Brasil vem seguindo essa tendência mundial e tem promulgado, desde a Constituição de 1988, várias normativas legais no sentido de promover a Educação Inclusiva. Dentre essas diretrizes, destaca-se a LBI, que garante para as pessoas com deficiência, dentre outros direitos, o acesso à comunicação e à informação, à não discriminação, à saúde, ao transporte e à educação. No tocante à educação, a LBI estabelece, no seu art. 27, a existência de um sistema educacional inclusivo para todos os níveis da vida escolar do aluno, com o objetivo de atingir o máximo desenvolvimento possível das habilidades físicas, sensoriais, intelectuais e sociais, levando em consideração seus interesses, características e necessidades de aprendizagem.

A LBI enfatiza ainda estratégias para melhoria da qualidade de vida e acesso ao trabalho para a pessoa com deficiência e a promoção da inclusão social. Dentre essas orientações, estão previstas ações para o desenvolvimento científico, a pesquisa e a inovação e a capacitação tecnológicas, voltadas à inclusão. Direcionando-se diretamente ao Direito à Educação, a LBI estabelece no seu artigo 28 que:

[...] incumbe ao poder público assegurar, criar, desenvolver, implementar, incentivar, acompanhar e avaliar: [...] VI pesquisas voltadas para o desenvolvimento de novos métodos e técnicas pedagógicas, de materiais didáticos, de equipamentos e de recursos de tecnologia assistiva; [...] (BRASIL, 2015).

Porém, mesmo com o estabelecimento dessas normativas que incentivam as pesquisas científicas para o desenvolvimento de novos recursos e serviços na área da Educação Inclusiva, elas ainda são consideradas escassas e isso acarreta consequências na elaboração das políticas públicas e destinação de investimentos para a área. Nesse sentido, Galvão Filho (2013), manifesta que:

[...] a escassez desses estudos acarreta, como uma de suas consequências mais importantes, grandes dificuldades para a definição e formatação de políticas públicas nessa área e para a configuração adequada de iniciativas de apoio e fomento a projetos com esse foco. (Galvão Filho, 2013, p. 6).

Ainda de acordo com Galvão Filho (2013), as pesquisas sobre Educação Inclusiva são parciais e provisórias, em função da inconsistência dos dados disponíveis e pelos avanços tecnológicos na área, devendo haver constante renovação e atualização.

Essa abordagem teórica teve o objetivo de atender ao objetivo específico desse estudo, apresentando as principais disposições legais acerca da Educação Inclusiva, focando nas disposições da LBI.

\section{Metodologia}

Para o desenvolvimento desse trabalho utilizamos a pesquisa bibliográfica e a pesquisa documental. No primeiro momento foi realizada a pesquisa bibliográfica para selecionar livros e artigos científicos para embasarem a construção da fundamentação teórica. De acordo com Gil (2010), a pesquisa bibliográfica é um trabalho de natureza exploratória, que propicia bases teóricas ao pesquisador para auxiliar no exercício reflexivo e crítico sobre o tema em estudo.

No segundo momento, realizamos uma pesquisa documental no Catálogo de Teses e Dissertações do IFTM com o propósito de levantar os dados necessários para responder ao objetivo geral do presente trabalho. A pesquisa documental, de acordo com Gil (2010), pode apresentar algumas vantagens por ser "fonte rica e estável de dados"; não implicando em altos custos e não exigindo contato direto com os sujeitos da pesquisa, possibilitando assim, uma leitura aprofundada das fontes. Ainda segundo Gil (2010), a pesquisa documental é semelhante à pesquisa bibliográfica, sendo a natureza das fontes o que as diferencia, ou seja, a pesquisa documental utiliza materiais que ainda não receberam tratamento analítico ou que ainda pode ser reelaborado de acordo com os objetivos da pesquisa. 
A pesquisa documental foi realizada utilizando como palavras-chave os termos "inclusão", "educação inclusiva" e “educação especial”. Nesse instrumento não utilizamos um recorte temporal para a pesquisa documental, sendo analisados todos os resultados apresentados. A coleta de dados e análise das informações seguiu a metodologia sugerida por Paiva, Silva e Gomes (2009), consistindo na montagem de planilhas com informações referentes aos autores e títulos de todos os textos, seguido do ano da publicação e do programa em que a pesquisa foi desenvolvida. Posteriormente foi realizada a leitura do resumo de cada pesquisa identificada com o objetivo de localizar informações referentes aos objetivos do trabalho.

A apresentação textual dos resultados desse trabalho é feita mediante exposição textual e gráfica em tabelas, com considerações acerca dos resultados encontrados, mediando os objetivos e considerações do universo pesquisado.

As atividades de buscas das dissertações disponíveis online, bem como a leitura e análise dos resumos das publicações foram efetuadas nos meses de janeiro, fevereiro e março de 2020.

\section{Resultados e Discussão}

Atualmente, o IFTM oferece cursos na modalidade stricto sensu nas seguintes áreas: Programa de Pós-graduação em Educação Profissional e Tecnológica - ProfEPT; Educação Tecnológica; Ciência e Tecnologia em Alimentos e Produção Vegetal.

Para a presente abordagem, inicialmente foi realizado acesso ao banco de dissertações e teses do IFTM $^{1}$, posteriormente foi adicionado o termo "inclusão" no campo de busca, retornando como resultado 08 dissertações distribuídas de acordo com o Quadro 1 abaixo, classificadas de acordo com o ano de realização do trabalho:

Quadro 1 - Resultados encontrados no banco de dissertações e teses do IFTM com o termo de busca "inclusão".

\begin{tabular}{|c|c|c|c|c|}
\hline Documento & Autor & Título & Ano & Programa \\
\hline 1. & $\begin{array}{l}\text { Maida Blandina Honório } \\
\text { Magalhães }\end{array}$ & $\begin{array}{l}\text { A manipulação de alimentos como alternativa } \\
\text { de inclusão profissional de deficientes visuais }\end{array}$ & 2014 & $\begin{array}{l}\text { Mestrado Profissional em } \\
\text { Ciência em Tecnologia de } \\
\text { Alimentos }\end{array}$ \\
\hline 2. & $\begin{array}{l}\text { Aparecida Rosário de } \\
\text { Oliveira Silva }\end{array}$ & $\begin{array}{l}\text { Educação inclusiva: tecnologia assistiva como } \\
\text { apoio à humanização de relações de ensino- } \\
\text { aprendizagem com crianças deficientes: } \\
\text { leitura de uma escola pública de Uberaba } \\
\text { (MG) }\end{array}$ & 2016 & $\begin{array}{l}\text { Mestrado Profissional em } \\
\text { Educação Tecnológica }\end{array}$ \\
\hline 3. & $\begin{array}{l}\text { Afranio Furtado de Oliveira } \\
\text { Neto }\end{array}$ & $\begin{array}{l}\text { Cotidiano: um software para auxiliar crianças } \\
\text { autistas a enfrentar os desafios diários }\end{array}$ & 2017 & $\begin{array}{l}\text { Mestrado Profissional em } \\
\text { Educação Tecnológica }\end{array}$ \\
\hline 4. & Rodolfo Bocardo Palis & $\begin{array}{l}\text { Narrativa de games: retextualização da } \\
\text { narrativa em jogos eletrônicos e suas } \\
\text { possibilidades educacionais }\end{array}$ & 2017 & $\begin{array}{l}\text { Mestrado Profissional em } \\
\text { Educação Tecnológica }\end{array}$ \\
\hline 5. & $\begin{array}{l}\text { Angélica Rosa de Oliveira } \\
\text { Fagundes }\end{array}$ & $\begin{array}{l}\text { Compostos bioativos e atividade antioxidante } \\
\text { em refrescos e sucos detox }\end{array}$ & 2018 & $\begin{array}{l}\text { Mestrado Profissional em } \\
\text { Ciência e Tecnologia de } \\
\text { Alimentos }\end{array}$ \\
\hline 6. & Pâmela Junqueira Freitas & $\begin{array}{l}\text { Os reflexos do programa de assistência } \\
\text { estudantil nos cursos superiores do IFTM } \\
\text { campus Patrocínio: }(2015-2016)\end{array}$ & 2018 & $\begin{array}{l}\text { Mestrado Profissional em } \\
\text { Educação Tecnológica }\end{array}$ \\
\hline 7. & Maria Djanira de Oliveira & $\begin{array}{l}\text { A inclusão no contexto dos institutos federais } \\
\text { de educação: a trajetória do NAPNE/IFTM- } \\
\text { Campus Uberaba }\end{array}$ & 2018 & $\begin{array}{l}\text { Mestrado Profissional em } \\
\text { Educação Tecnológica }\end{array}$ \\
\hline 8. & $\begin{array}{l}\text { Marco Antônio Manzan } \\
\text { Veludo }\end{array}$ & $\begin{array}{l}\text { Google na sala de aula: aplicado para discentes } \\
\text { do ensino fundamental de uma escola } \\
\text { particular de Uberaba- MG }\end{array}$ & 2018 & $\begin{array}{l}\text { Mestrado Profissional em } \\
\text { Educação Tecnológica }\end{array}$ \\
\hline
\end{tabular}

Fonte: Banco de dissertações e teses do IFTM (2020).

\footnotetext{
${ }^{1}$ Informação obtida através do endereço eletrônico https://iftm.edu.br/bdt/.
} 
No segundo momento, a busca foi retomada com o termo "educação inclusiva" retornando como resultados duas dissertações já apresentadas na primeira busca, demonstradas no Quadro 2, classificadas de acordo com o ano de realização dos trabalhos:

Quadro 2 - Resultados encontrados no banco de dissertações e teses do IFTM com o termo de busca "educação inclusiva".

\begin{tabular}{|c|l|l|l|l|}
\hline Documento & \multicolumn{1}{|c|}{ Autor } & \multicolumn{1}{|c|}{ Título } & \multicolumn{1}{|c|}{ Ano } & \multicolumn{1}{c|}{ Programa } \\
\hline 1. & Aparecida Rosário de Oliveira & $\begin{array}{l}\text { Educação inclusiva: tecnologia assistiva } \\
\text { como apoio à humanização de relações de } \\
\text { ensino-aprendizagem com crianças } \\
\text { deficientes: leitura de uma escola pública } \\
\text { de Uberaba (MG) }\end{array}$ & $\begin{array}{l}\text { Mestrado Profissional em } \\
\text { Educação Tecnológica }\end{array}$ \\
\hline 2. & Maria Djanira de Oliveira & $\begin{array}{l}\text { A inclusão no contexto dos institutos } \\
\text { federais de educação: a trajetória do } \\
\text { NAPNE/IFTM- Campus Uberaba }\end{array}$ & $\begin{array}{l}\text { Mestrado Profissional em } \\
\text { Educação Tecnológica }\end{array}$ \\
\hline
\end{tabular}

Fonte: Banco de dissertações e teses do IFTM (2020).

Através da exposição do quadro 2, pode-se observar que as dissertações encontradas apresentam pesquisas referentes a utilização da tecnologia assistiva na educação inclusiva e ao trabalho do NAPNE realizado no Campus Uberaba.

No terceiro momento utilizou-se o termo “educação especial” para busca, retornando dois resultados, 01 dissertação já citada nos quadros 1 e 2 e 01 nova dissertação, ambas do ano de 2016, expostas no Quadro 3:

Quadro 3 - Resultados encontrados no banco de dissertações e teses do IFTM com o termo de busca "educação especial".

\begin{tabular}{|c|l|l|l|l|}
\hline Documento & \multicolumn{1}{|c|}{ Autor } & \multicolumn{1}{c|}{ Título } & \multicolumn{1}{c|}{ Ano } & \multicolumn{1}{c|}{ Programa } \\
\hline 1 & $\begin{array}{l}\text { Carolina Andrade Rodrigues da } \\
\text { Cunha }\end{array}$ & $\begin{array}{l}\text { Língua espanhola na educação } \\
\text { brasileira: trajetória e análise das } \\
\text { concepções de ensino }\end{array}$ & Educação Tecnológica \\
\hline 2 & Aparecida Rosário de Oliveira Silva & $\begin{array}{l}\text { Educação inclusiva: tecnologia } \\
\text { assistiva como apoio à humanização } \\
\text { de relações de ensino-aprendizagem } \\
\text { com crianças deficientes: leitura de } \\
\text { uma escola pública de Uberaba (MG) }\end{array}$ & $\begin{array}{l}\text { Mestrado Profissional em } \\
\text { Educação Tecnológica }\end{array}$ \\
\hline
\end{tabular}

Fonte: Banco de dissertações e teses do IFTM (2020).

Segundo o Quadro 3, observa-se que a nova dissertação encontrada versa sobre uma pesquisa referente à formação de professores de Língua Espanhola, com foco no percurso dos métodos utilizados por professores de espanhol.

Após a leitura dos resumos das dissertações recuperadas, descartamos os trabalhos de Cunha (2016), Palis (2017), Fagundes (2018), Freitas (2018) e Veludo (2018) por tratarem respectivamente de inclusão social proporcionada pelo domínio em um segundo idioma; inclusão de interfaces de jogos eletrônicos e seu uso pelos jovens na aquisição do conhecimento; inclusão de frutas e hortaliças na dieta; benefícios dos Programas de Assistência estudantil e inclusão no ensino a distância através da utilização de ferramenta específica. De acordo com os objetivos da presente proposta, ou seja, identificar pesquisas sobre a inclusão na educação e seu conjunto de ferramentas e estratégias para a inserção de indivíduos com qualquer tipo de deficiência dentro do ambiente escolar regular, foram selecionadas, 04 dissertações para continuidade da investigação, classificadas por ano de realização do trabalho e expostas no Quadro 4. 
Quadro 4 - Dissertações encontradas no banco de dissertações e teses do IFTM selecionadas para a pesquisa:

\begin{tabular}{|c|l|l|l|l|}
\hline Documento & \multicolumn{1}{|c|}{ Autor } & \multicolumn{1}{|c|}{ Título } & \multicolumn{1}{|c|}{ Ano } & \multicolumn{1}{|c|}{ Programa } \\
\hline 1. & $\begin{array}{l}\text { Maida Blandina Honório } \\
\text { Magalhães }\end{array}$ & $\begin{array}{l}\text { A manipulação de alimentos como } \\
\text { alternativa de inclusão profissional de } \\
\text { deficientes visuais }\end{array}$ & $\begin{array}{l}\text { Mestrado Profissional em } \\
\text { Ciência em Tecnologia de } \\
\text { Alimentos }\end{array}$ \\
\hline 2. & $\begin{array}{l}\text { Aparecida Rosário de Oliveira } \\
\text { Silva }\end{array}$ & $\begin{array}{l}\text { Educação inclusiva: tecnologia assistiva } \\
\text { como apoio à humanização de relações } \\
\text { de ensino-aprendizagem com crianças } \\
\text { deficientes: leitura de uma escola } \\
\text { pública de Uberaba (MG) }\end{array}$ & $\begin{array}{l}\text { Mestrado Profissional em } \\
\text { Educação Tecnológica }\end{array}$ \\
\hline 3. & Afranio Furtado de Oliveira Neto & $\begin{array}{l}\text { Cotidiano: um software para auxiliar } \\
\text { crianças autistas a enfrentar os desafios } \\
\text { diários }\end{array}$ & $\begin{array}{l}\text { A inclusão no contexto dos institutos } \\
\text { federais de educação: a trajetória do } \\
\text { NAPNE/IFTM- Campus Uberaba }\end{array}$ & $\begin{array}{l}\text { Mestrado Profissional em } \\
\text { Educação Tecnológica }\end{array}$ \\
\hline 4. & Maria Djanira de Oliveira & Educação Tecnológica \\
\hline
\end{tabular}

Fonte: Banco de dissertações e teses do IFTM (2020).

Conforme exposto no Quadro 4, as dissertações selecionadas foram concluídas nos anos de 2014; 2016 ; 2017 e 2018 respectivamente.

Atendendo ao objetivo específico do presente estudo, identificou-se que a maioria das pesquisas foram desenvolvidas no Mestrado Profissional em Educação Tecnológica (PROFEPT), que tem como objetivo proporcionar formação em educação profissional e tecnológica aos profissionais da Rede Federal de Educação Profissional, Científica e Tecnológica (RFEPCT), visando tanto a produção de conhecimento quanto o desenvolvimento de produtos, por meio da realização de pesquisas que integrem os saberes inerentes ao mundo do trabalho e ao conhecimento sistematizado. (MEC, 2019).

De acordo com o proposto no objetivo geral desse trabalho, nas pesquisas selecionadas foram analisados os objetivos e os principais resultados. Sendo assim, identificou-se que a proposta de estudo da pesquisadora Magalhães (2014) foi focada na inclusão social e profissional, baseando-se na promoção de treinamentos em manipulação de alimentos para pessoas com deficiência visual (cegos e de baixa visão) possibilitando a inclusão de cidadãos com deficiência no mercado de trabalho em Unidades de Alimentação e Nutrição como manipuladores de alimentos, através do desenvolvimento de ferramentas pedagógicas adaptadas.

Em termos práticos, a adequação no ensino para cegos deve incluir várias possibilidades, entre elas: sistema braile e material adaptado em relevo; para alunos de baixa visão: recurso óptico necessário, lupas, computador com sintetizador de voz, softwares educativos específicos e iluminação adequada (Aranha, 2005).

A LBI também incentiva a formação para o trabalho, bem como a habilitação e a reabilitação profissional, na seção II - Da Habilitação Profissional e Reabilitação Profissional, art. 36, quando expressa que o poder público deve implementar serviços e programas completos de habilitação profissional e de reabilitação profissional para que a pessoa com deficiência possa ingressar, continuar ou retornar ao campo do trabalho, respeitados sua livre escolha, sua vocação e seu interesse (BRASIL, 2015).

Após a realização de treinamentos e o desenvolvimento de ferramentas pedagógicas concluiu-se que a inclusão profissional de alunos cegos e de baixa visão é possível na área de manipulação de alimentos desde que sejam utilizados todos os recursos que contribuam para essa abordagem. (Magalhães, 2014).

Já a pesquisadora Silva (2016) analisou a contribuição das Tecnologias de Informação e Comunicação (TIC) para a aprendizagem e, como principal resultado do estudo, foi reforçada a necessidade de formação atualizada dos professores de acordo com as inovações tecnológicas disponíveis para utilização, fato também explicitado por Valente (1999, p. 19), “a questão da formação do professor mostra-se de fundamental importância no processo de introdução da informática na educação, exigindo 
soluções inovadoras e novas abordagens que fundamentam os cursos de formação". A pesquisadora Silva (2016) pontuou ainda a importância da elaboração e acompanhamento de planejamentos diversificados, de acordo com a necessidade educacional do indivíduo e a classificação da tecnologia assistiva como um ponto de apoio e reforço na aprendizagem.

A tecnologia assistiva também foi citada no estudo realizado pelo pesquisador Oliveira Neto (2017) que focou no desenvolvimento de um aplicativo móvel, para auxiliar crianças autistas em seu cotidiano, levando em consideração os tipos de perturbações e as intervenções educacionais existentes nas tecnologias de apoio, que serviram de base para a concepção do aplicativo. De acordo com Cook e Polgar (2008), a tecnologia assistiva, também denominada como tecnologia de apoio é qualquer serviço ou ferramenta adaptativa, que ajuda um indivíduo com deficiência, em suas atividades diárias. Ainda para os citados autores, existem relatos de pessoas com deficiência que utilizaram essa tecnologia e se tornaram e permaneceram independentes.

Como resultado do trabalho de Oliveira Neto (2017) foi desenvolvido um software que foi testado por uma equipe multidisciplinar e retornou feedback positivo, mencionando que caso o aplicativo seja adotado por crianças autistas, poderá ajudar no tratamento, auxiliando aos pais na organização do cotidiano das crianças e essa organização ajuda a diminuir a ansiedade levando a criança a ter uma melhor qualidade de vida e, com o tempo, quem sabe até uma maior inclusão social (Oliveira Neto, 2017).

A pesquisadora Oliveira (2018) pautou seu trabalho em apresentar um importante programa para a inclusão adotado pelo IFTM, o Núcleo de Atendimento às Pessoas com Necessidades Educacionais Específicas (NAPNE). A proposta foi contextualizar os processos de inclusão nos Institutos Federais sob a perspectiva do NAPNE com foco no Campus Uberaba, por meio de análise documental do núcleo, das políticas públicas e da formação dos professores para educação inclusiva (OLIVEIRA, 2018). O estudo apresentou ainda resultados de pesquisas anteriores sobre inclusão nos Institutos Federais do Rio Grande do Sul, do Rio de Janeiro e do Amazonas. Como maior desafio para a obtenção dos objetivos da Política Nacional de Educação Especial na perspectiva da Educação foi citada a formação continuada de professores para atuar no Atendimento Educacional Especializado (AEE) e assim garantir a inclusão do aluno com deficiência, Transtornos Globais do Desenvolvimento (TGD) e altas habilidades / superdotação no ensino regular.

O professor tem que ter uma formação que o ajude a atender essa clientela, com a formação que temos hoje é praticamente impossível. Temos medo e por isso negamos a presença desses alunos dentro das nossas escolas. O professor que tiver uma formação terá uma clareza que o ajudará, não existem metodologias para atender essa clientela, existem recursos tecnológicos, por exemplo, para resolver o problema de um aluno de baixa visão e a escola dispor de recursos que o auxiliem no aprendizado, o problema dele estará resolvido. Se para um aluno com surdez, a escola dispor de material visual suficiente para ele aprender, ele conseguirá aprender a ler, a escrever e é claro, aprende de uma forma diferente das pessoas que ouvem. O professor tem que estar preparado para entender isso, ao corrigir, por exemplo, a redação de um aluno que é surdo o professor de português não poderá utilizar o mesmo critério que utiliza para corrigir a redação de um aluno que é ouvinte. (Pontes, 2006, p. 36).

Como podemos verificar diante dos resultados, o número de pesquisas encontradas sobre o tema foi baixo, sendo a maioria realizada no programa de Mestrado Profissional em Educação Tecnológica. Esse número reduzido de estudos sobre a Educação Inclusiva reflete a escassez já citada por Galvão Filho (2013) e suas implicações como dificuldades na formulação e acompanhamento das políticas públicas sobre o tema além de perda de possíveis investimentos na área.

\section{Considerações Finais}

O desenvolvimento do presente estudo possibilitou identificar pesquisas sobre educação inclusiva já desenvolvidas em programas de Pós-graduação Stricto sensu do IFTM, também permitiu conhecer as principais indicações da LBI acerca do tema e suas aplicações práticas em pesquisas realizadas no IFTM. 
Após a leitura das dissertações selecionadas foi possível identificar a relevância da formação continuada para os profissionais da área da Educação Inclusiva. Também foi evidenciado que alguns trabalhos buscam descrever operações e métodos desenvolvidos profissionalmente pelos pesquisadores. Essa familiaridade com as ações enriquece o trabalho, pois permite a exteriorização de percepções vivenciadas de forma direta. Outro fator mencionado em todas as pesquisas analisadas é a utilização da tecnologia assistiva a favor do ensino, suas possibilidades e os desafios na sua utilização.

Portanto, dada a importância do assunto e o baixo número de estudos na área, torna-se necessário o incentivo de novas pesquisas, bem como o acompanhamento dos trabalhos já desenvolvidos, pois ficou comprovado que as experiências socializadas exprimem estratégias para o alcance dos objetivos da Educação Inclusiva.

\section{Referências}

Aranha, M. S. F. (2005). Educação Inclusiva: a escola. Secretaria da Educação Especial.

Cook, A. M.; Polgar, J. M. (2008). Cook \& Hussey’s Assistive Technologies: Principles And Practice. Mosby Elsevier.

Cunha, C. A. R. da. (2016). Língua espanhola na educação brasileira: trajetória e análise das concepções de ensino. Dissertação (Mestrado Profissional em Educação Tecnológica) - IFTM.

Fagundes, A. R. de O. (2018). Compostos bioativos e atividade antioxidante em refrescos e sucos detox. Dissertação (Mestrado Profissional em Ciência e Tecnologia de Alimentos) - IFTM.

Freitas, P. J. (2018). Os reflexos do programa de assistência estudantil nos cursos superiores do IFTM campus Patrocínio: (2015-2016). Dissertação (Mestrado Profissional em Educação Tecnológica) - IFTM.

Galvão Filho, T.A. (2013). Tecnologia Assistiva e Educação. In. Souza, R. de C., Barbosa, J.S.L. Educação Inclusiva, tecnologia e tecnologia assistiva. Criação. Gil, A.C. (2010). Como elaborar projetos de pesquisa. Atlas.

Gonçalves, F. S. (2018). Os desafios atuais para a implementação da Ações Afirmativas nas Instituições de Educação Superior: Quem são os sujeitos de direitos em um contexto de fraudes? In: ENPOS UFPEL. UFPEL.

Lei no 13.146 de 06 de julho de 2015. (2015). Brasília.

Magalhães, M. B. H. (2014). A manipulação de alimentos como alternativa de inclusão profissional de deficientes visuais. Dissertação (Mestrado Profissional em Ciência em Tecnologia de Alimentos) - IFTM.

Mantoan, M.T.E. (2004). O direito de ser, sendo diferente na escola. Revista Conselho da Justiça Federal/Centro de Estudos Judiciários da Justiça Federal.

Menezes, P. D. L. de. (2015). Reflexões sobre o Sistema de Cotas no Brasil. Universidade Federal da Paraíba.

Oliveira Neto, A. F. de. (2017). Cotidiano: um software para auxiliar crianças autistas a enfrentar os desafios diários. Dissertação (Mestrado Profissional em Educação Tecnológica) - IFTM.

Oliveira, M. D. de. (2018). A inclusão no contexto dos institutos federais de educação: a trajetória do NAPNE/IFTM- Campus Uberaba. Dissertação (Mestrado Profissional em Educação Tecnológica) - IFTM.

Paiva, V. L. M. O.; Silva, M. M.; Gomes, I. F. (2009). Sessenta anos de Linguística Aplicada: de onde viemos e para onde vamos. In: Pereira, R. C.; Roca, P. Linguística aplicada: um caminho com diferentes acessos. Contexto.

Palis, R. B. (2017). Narrativa de games: retextualização da narrativa em jogos eletrônicos e suas possibilidades educacionais. Dissertação (Mestrado Profissional em Educação Tecnológica) - IFTM.

PNAES - Plano Nacional de Assistência Estudantil. Ministério da Educação. 2010. http://portal.mec.gov.br/pnaes.

Pontes, J. (2005). Programa TEC NEP - Educação, Tecnologia e Profissionalização para Pessoas com Necessidades Educacionais Especiais. In: Seminário Nacional.

PROEPT - Objetivos do Programa de Mestrado Profissional em Educação Tecnológica. 2019. http://portal.mec.gov.br.

Santos, W. (2011). Política de cotas para pessoas com deficiência; mitigação ao princípio da isonomia? Revista de Informação Legislativa.

Silva, A. R. de O. (2016). Educação inclusiva: tecnologia assistiva como apoio à humanização de relações de ensino-aprendizagem com crianças deficientes: leitura de uma escola pública de Uberaba (MG). Dissertação (Mestrado Profissional em Educação Tecnológica) - IFTM.

Valente, J. A. (Org). (1999). O computador na sociedade do conhecimento. UNICAMP/NIED.

Veludo, M. A. M. (2018). Google sala de aula: aplicado para discentes do ensino fundamental de uma escola particular de Uberaba- MG. Dissertação (Mestrado Profissional em Educação Tecnológica) - IFTM. 